Methods Children presenting to the acute care team in a tertiary PED who had blood tests over a 2 -week period in April 2019 were enrolled. Blood tests requested were interpreted in line with presenting features and clinical impression. Sequel to the evaluation, anonymised case vignettes were circulated to trainees and advanced nurse practitioners (ANPs) to ascertain their practice of blood tests requests. Recurrent audit cycles have been ongoing for sustainability of scientific logic based practice.

Results One hundred and one children who had blood tests were enrolled. All the children had full blood count test done; 93\%, 70\%, 47\%, 44\% and 32\% had renal function, liver bloods, bone profile, blood culture and clotting testing done respectively. Over half of these blood tests had no clear clinical indication. The yield of the tests performed without clinical indication was $0 \%$. Case vignettes were attempted by 26 trainees and ANPs to evaluate their attitudes to blood test requests. Number of requested blood tests not clinically indicated was lower than anticipated; probably explained by selfthought processes. Repeat cycles have shown over $80 \%$ reduction in number of liver blood tests performed in jaundiced infants.

Conclusions The utility of blood tests may be an easily forgotten subject in paediatric training. It is noteworthy that implementation of measures to reduce unnecessary blood test requests will result in an estimated savings of $£ 1000 /$ month. Interventions comprising regular educational sessions, audits, posters and blood tests champions are in place to sustain the reduction of unnecessary blood tests.

\section{G239(P) USE OF NON-INVASIVE RESPIRATORY SUPPORT IN CHILDREN REFERRED TO A PAEDIATRIC INTENSIVE CARE RETRIEVAL SERVICE}

M Emedo, P Ramnarayan. Children's Acute Transport Service (CATS), Great Ormond Street Hospital, London, UK

\subsection{6/archdischild-2020-rcpch.206}

Background High flow nasal cannula (HFNC) is now a widely used mode of Non Invasive Respiratory Support (NRS) for children with respiratory distress.

Objectives To describe the trends in use of NRS for children referred to a paediatric intensive care transport service, and identify any associations between mode used and final retrieval status.

Methods Retrospective review of two grouped cohorts to capture any seasonal variability; referrals from December and May of 2012/2013 were compared with 2017/2018. Children on acute BIPAP/CPAP or HFNC were included for analysis. Ventilation mode, proportions retrieved and intubation rates were compared, as were demographics and indicators of disease severity.

Results In total 204 children were referred on NRS, most commonly with bronchiolitis, pneumonia or cardiac conditions.

There was no significant difference in baseline disease severity between the cohorts, as determined by SpO2:FiO2 ratio $(\mathrm{p}=0.07)$ or referrer rated respiratory distress. In the $2017 / 2018$ time periods, $30 \%$ of all referrals received by the service were on NRS vs. $15 \%$ in 2012/2013. Of these NRS referrals, HFNC represented a larger proportion in 2017/2018 than in $2012 / 2013$ (57 vs $17 \%$ ).
There was no significant difference in final retrieval status or rates of intubation for transfer according to mode of NRS used at time of referral $(\mathrm{p}=0.57)$.

Conclusion In 2017/2018 NRS was used more frequently for children referred for retrieval, and HFNC was more commonly chosen to deliver this compared with 2012/ 2013. This rise in popularity of HFNC has not been associated with a larger proportion of referrals requiring retrieval, or more children eventually needing intubation for transport.

\section{G240(P) ULTRASOUND AFTER UTI: IS IT WORTH BEING EXTRA NICE?}

AJ Smith, B Stanhope. Emergency Department, Birmingham Women's and Children's NHS FT, Birmingham, UK

\subsection{6/archdischild-2020-rcpch.207}

Background Our Paediatric Emergency Department (ED) follows a local, multi-specialty designed guideline for the investigation of Urinary Tract Infection (UTI). All children and young people (CYP) presenting with a first proven, or otherwise previously uninvestigated, UTI undergo routine outpatient urinary tract ultrasound (US) imaging. This pathway deviates from National Institute for Health and Care Excellence (NICE) guidance which recommends that children over six months old only undergo imaging if their UTI is atypical or recurrent. The primary aim of this project was to establish the proportion of those undergoing imaging who had a urinary tract abnormality (pre-existing or resultant) requiring specialist referral. We then appraised whether the yield from our investigation pathway justified deviation from NICE guidance.

Method A retrospective service evaluation was conducted for the period 1 May 2016 to 30 April 2019. Inclusion criteria: all ED-requested US for patients aged 6 months and over with a documented first proven or previously uninvestigated UTI. Exclusion criteria: CYP under six months old; duplicate requests; previous US for UTI; pre-existing UTI follow-up; known pre-existing nephro-urological condition; US indication unrelated to UTI. Patients were identified using the Trust radiology system, with a review of the ED documentation and radiology reports. Reported abnormalities were reviewed against our guideline's agreed criteria for specialist referral, to determine if follow-up planning was appropriate.

Results 358 Urinary Tract US were requested during the study period, 161 of which were requested in line with the local UTI guideline. 5 (3.1\%) US were not attended but were included in an intention to investigate analysis. 35 (21.7\%) patients had nephro-urological abnormalities identified that were referred for general paediatric, urology or nephrology follow-up. An additional 15 (9.3\%) patients had US findings that may also have warranted nephro-urological follow-up, giving a potential $50(31.1 \%)$ patients with abnormalities detected outside of NICE guidance.

Conclusion Up to $31 \%$ of patients investigated with US during the three year study period had an abnormality detected that would have remained unidentified if practicing within NICE guidance. Routine US offers a non-invasive, low-risk, low-cost investigative tool to identify nephro-urological abnormalities earlier, improving safety and quality of patient care. 